\title{
Effect of symmetry reduction on the electronic transitions in polytypic $\mathrm{GdAl}_{3}\left(\mathrm{BO}_{3}\right)_{4}: \mathrm{Eu}: \mathrm{Tb}$ crystals
}

\author{
$\underline{\text { K. Lengyel }}^{{ }^{*}}{ }^{*}$, E. Beregi ${ }^{1}$, I. Földvári ${ }^{1}$, G. Corradi ${ }^{1}$, L. Kovács ${ }^{1}$, P. Solarz ${ }^{2}$, W. Ryba- \\ Romanowski $^{2}$
}

${ }^{1}$ Institute for Solid State Physics and Optics, Wigner Research Centre for Physics, Hungarian Academy of Sciences, Budapest, Hungary

${ }^{2}$ Institute of Low Temperature and Structure Research, Polish Academy of Sciences, Wrocław, Poland

*Corresponding author: lengyel.krisztian@wigner.mta.hu

\begin{abstract}
The existence of a recently described monoclinic phase $(C 2 / c, \mathrm{Z}=8)$ [1] in addition to the well-known Huntite type rhombohedral (R32) polytypic modification of the $\mathrm{GdAl}_{3}\left(\mathrm{BO}_{3}\right)_{4}$ (GAB) crystal at room temperature provides a unique possibility to investigate the incorporation of rare earth dopants into slightly modified crystal lattice by spectroscopic methods. In these characteristic GAB structures the dopant ions, e.g $\mathrm{Tb}^{3+}$ or $\mathrm{Eu}^{3+}$, possess slightly different neighbor geometries and local symmetries. The $\mathrm{Tb}^{3+}:{ }^{7} \mathrm{~F}_{6} \rightarrow{ }^{5} \mathrm{D}_{4}$ and $\mathrm{Eu}^{3+}$ : ${ }^{7} \mathrm{~F}_{0,1,2} \rightarrow{ }^{5} \mathrm{D}_{0,1,2}$ electronic transitions were successfully identified in the absorption spectra using polarization, concentration and temperature dependent measurements in both polytypic modifications. The positions of the investigated $\mathrm{Tb}$ lines are shifted by up to $10 \mathrm{~cm}^{-1}$ due to symmetry changes. In addition, some of the Eu lines show splittings of about $4-30 \mathrm{~cm}^{-1}$ as a consequence of the change of the local environment. From the room temperature absorption measurements some of the low energy crystal field levels of ${ }^{7} \mathrm{~F}$ and ${ }^{5} \mathrm{D}$ states of the $\mathrm{Eu}^{3+}$ ions were successfully determined for both modifications.
\end{abstract}

Keywords: electronic transition, absorption, polytypism, GdA13(BO3)4, Europium

\section{Introduction}

The huntite type aluminium borate crystals $\left(\mathrm{RAl}_{3}\left(\mathrm{BO}_{3}\right)_{4}\right.$, where $\mathrm{R}$ denotes rare earth elements including yttrium), have been investigated for a long time since the members of this crystal family exhibit strong chemical stability, outstanding physical and optical properties, such as 
wide optical transmission range in the ultraviolet [1-3]. They are excellent hosts for laser active rare earths, since the substitution of these ions at the trigonal prism sites usually occurs without charge compensation and lattice distortion. The efficiency of the borate-based light sources depends in a complex way on the active rare earth ions present. Enhanced energy transfer rate was observed in the visible range luminescence of the $\mathrm{Eu}$ and $\mathrm{Tb}$ co-doped $\mathrm{GdAl}_{3}\left(\mathrm{BO}_{3}\right)_{4}(\mathrm{GAB})$ crystal after excitation the $\mathrm{Gd}^{3+}$ host ions in the VUV range [5,6]. In addition, the ${ }^{7} \mathrm{~F}_{2}-{ }^{5} \mathrm{D}_{0}$ transition of $\mathrm{Eu}^{3+}$ ion in the huntite type GAB crystal does not follow strictly the selection rules for electrical dipole transition $[7,8]$. This effect has generated a series of detailed spectroscopic measurements and crystal field calculations [9-13] showing the induced electric dipole character of the ${ }^{7} \mathrm{~F}_{2-}{ }^{5} \mathrm{D}_{0}$ transition [9].

Although borate crystals are most useful for optical applications if possessing the huntite structure $(R 32)$ it has become clear that they could be grown in polytypic modifications with different symmetries (e.g. $C 2, C 2 / c[4,14])$. Growth temperature and cooling rate were found to be important factors for producing the given polytypic modifications of these borate crystals. The possibility and realization of polytypic modifications in borate crystals has been investigated by Belokoneva and Timchenko in detail [15]. They have shown that the observed modifications can be explained in order-disorder theory by different arrangements of composition layers common for all polytypes and corresponding to the 'ab' plane of the monoclinic structures. In the unified model $[15,16]$ several possibilities of the polytypic packing were predicted. Some of those structures have already been experienced in various borate crystals, while others could not be produced so far. One of the missing structures with $C 2 / c$ space group with a unit cell consisting of 8 molecules has recently been prepared [1]. The emergence of polytypic phases as a function of the starting crystallization temperature was demonstrated in Eu and $\mathrm{Tb}$ co-doped GAB crystals grown by the high temperature top seeded solution growth (HTTSSG) method. Both the rhombohedral (R32) and the monoclinic $(C 2 / c, \mathrm{Z}=8)$ phases could be separately prepared with the same composition [1]. The dopant rare earth ions enter Gd sites in both cases, however their local site symmetry is lowered from trigonal to $\mathrm{C} 2$ in the monoclinic phase [1].

In this work the results of spectroscopic measurements of $\mathrm{Tb}$ and Eu doped GAB crystals with monoclinic $(C 2 / c, \mathrm{Z}=8)$ structure will be presented for the first time and compared to the case of the rhombohedral (R32) modification. The temperature, concentration and polarization dependences of the rare earth transition bands were investigated and the term structures of the low energy parts of ${ }^{7} \mathrm{~F}$ and ${ }^{5} \mathrm{D}$ states of the $\mathrm{Eu}^{3+}$ ions were determined and compared for the different symmetries. 


\section{Experimental}

The investigated GAB single crystals were grown by the HTTSSG method from a $\mathrm{K}_{2} \mathrm{Mo}_{3} \mathrm{O}_{10}: \mathrm{B}_{2} \mathrm{O}_{3}$ flux (see details in [17]). The starting concentrations of the dopants in the solution are listed in Table 1. The structure of the as-grown crystals was identified by X-ray diffraction (XRD) measurements. Crystal samples were cut, oriented and polished to obtain optical quality.

\begin{tabular}{|c|c|c|c|}
\hline No. & $\mathrm{Eu} \mathrm{[mol \% ]}$ & $\mathrm{Tb}[\mathrm{mol} \%]$ & Symmetry \\
\hline 1 & 0 & 5 & $R 32$ \\
\hline 2 & 0 & 10 & $R 32$ \\
\hline 3 & 0 & 20 & $R 32$ \\
\hline 4 & 20 & 20 & $R 32$ \\
\hline 5 & 20 & 20 & $C 2 / c$ \\
\hline
\end{tabular}

Table 1. The concentrations of $\mathrm{Tb}^{3+}$ and $\mathrm{Eu}^{3+}$ ions in the starting solution and the space groups of the as-grown GAB crystals.

The absorption spectra were recorded by a BRUKER IFS 66/v FTIR spectrophotometer with a resolution at about $0.2 \mathrm{~cm}^{-1}$. For the polarization dependent measurements an UV-visible polymer polarizer was used. Temperature dependent measurements in the range of 9-300 K were carried out in a closed-cycle helium cryostat.

\section{Results and discussion}

The huntite type borates are uniaxial optical crystals with the optical axis coinciding with the trigonal axis, while the monoclinic modifications are biaxial. The selection rules for the polarization dependent measurements are summarized in Table $2[18,19]$. The electric or magnetic dipole behavior of the transitions have been shown by white and grey background of the columns, respectively. 


\begin{tabular}{|c|c|c|c|c|c|c|}
\hline $\mathrm{D}_{3}$ & \multicolumn{2}{|c|}{$\mathrm{A} 1$} & \multicolumn{2}{c|}{$\mathrm{A} 2$} & \multicolumn{2}{c|}{$\mathrm{E}$} \\
\hline $\mathrm{A} 1$ & - & - & $\pi$ & $\sigma$ & $\alpha \sigma$ & $\alpha \pi$ \\
\hline $\mathrm{A} 2$ & $\pi$ & $\sigma$ & - & - & $\alpha \sigma$ & $\alpha \pi$ \\
\hline $\mathrm{E}$ & $\alpha \sigma$ & $\alpha \pi$ & $\alpha \sigma$ & $\alpha \pi$ & $\alpha \pi \sigma$ & $\alpha \pi \sigma$ \\
\hline
\end{tabular}

\begin{tabular}{|c|c|c|c|c|}
\hline $\mathrm{C}_{2}$ & \multicolumn{2}{|c|}{$\mathrm{A}$} & \multicolumn{2}{c|}{$\mathrm{B}$} \\
\hline $\mathrm{A}$ & $\mathrm{z}$ & $\mathrm{Rz}$ & $\mathrm{x}, \mathrm{y}$ & $\mathrm{Rx}, \mathrm{Ry}$ \\
\hline $\mathrm{B}$ & $\mathrm{x}, \mathrm{y}$ & $\mathrm{Rx}, \mathrm{Ry}$ & $\mathrm{z}$ & $\mathrm{Rz}$ \\
\hline
\end{tabular}

Table 2. Selection rules for the polarization directions for both symmetry modifications (a: trigonal $\mathrm{D}_{3}$, b: monoclinic $\mathrm{C}_{2}$ ). The background of the cell is white or grey when the transition has electric or magnetic dipole behavior, respectively.

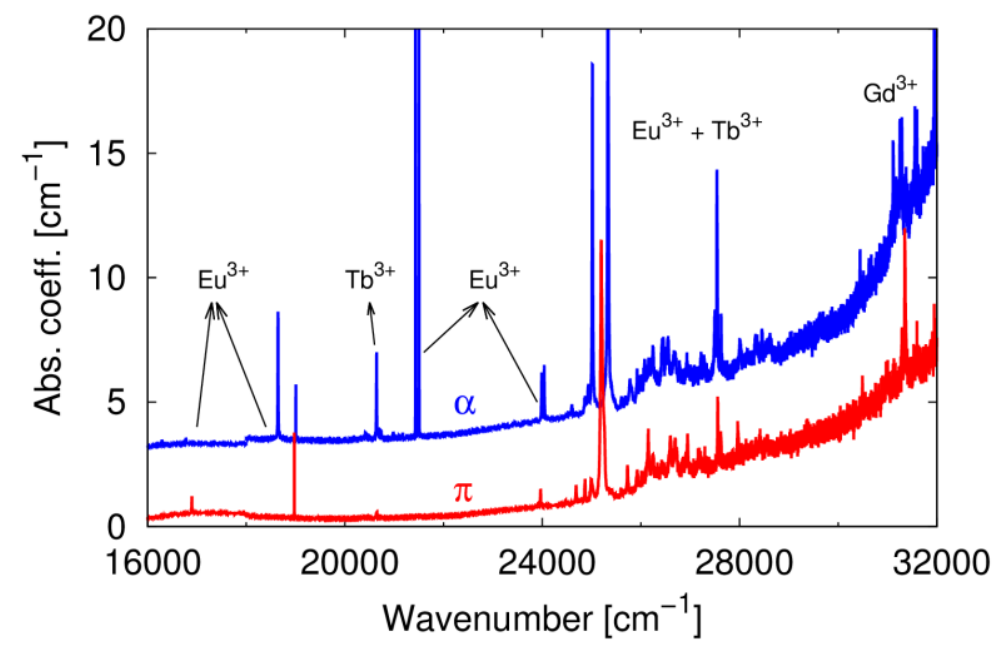

Fig. 1. Visible-UV absorption spectra of the rhombohedral GAB:Tb,Eu (No. 4) crystal measured in the $\alpha$ and $\pi$ polarizations at room temperature.

Several absorption bands corresponding to the electronic transitions of $\mathrm{Tb}^{3+}, \mathrm{Eu}^{3+}$ and $\mathrm{Gd}^{3+}$ ions can be seen in the absorption spectra measured in the $16000-32000 \mathrm{~cm}^{-1}$ range showing strong polarization dependence (see Fig. 1). Transitions up to $22000 \mathrm{~cm}^{-1}$ wavenumber can be clearly distinguished, however, above that the absorption bands overlap each other. Since the electronic transitions of $\mathrm{Gd}^{3+}$ ions are situated above $31500 \mathrm{~cm}^{-1}$, only the transitions of $\mathrm{Tb}^{3+}$ and $\mathrm{Eu}^{3+}$ ions have been studied in this work.

As was shown in ref. [1] a pseudo-trigonal axis behaving as a pseudo-optical axis can also be defined in the monoclinic GAB crystal. With this simplification the results of the polarization measurements can be treated in a similar way both for trigonal and monoclinic modifications using the following notations. In the case of rhombohedral GAB crystals for light propagating 
perpendicular to the optical axis the $\pi_{\mathrm{r}}$ and $\sigma_{\mathrm{r}}$ polarizations mean electric fields parallel and perpendicular to the $c$ axis, respectively, while the $\alpha_{\mathrm{r}}$ symbol is used for light propagation coinciding with the optical axis. In the monoclinic case one of the dielectric axes coincides with the twofold axis (z), the second one with the pseudo-trigonal axis (x) and the third one (y) is perpendicular to those two. Samples with surfaces perpendicular and parallel to the pseudo-trigonal axis were prepared for comparison. However, due to complex growth and morphological reasons the normal of the surface parallel to the pseudo-trigonal axis has an angle of $60^{\circ}$ to the z-axis. Accordingly, the $\pi_{\mathrm{m}}$ and $\sigma_{\mathrm{m}}$ symbols are used for light propagating perpendicular to the pseudo-trigonal axis whereby the polarization is parallel $\left(E_{x}\right)$ and perpendicular $\left(E_{y}+E_{z}\right)$ to the $x$ axis, respectively (i.e. for the case of the sample cut parallel to psudo-trigonal axis). The $\alpha_{\mathrm{r}}$ symbol is used for light propagating along the pseudo-trigonal $\mathrm{x}$ axis where the $\mathrm{E}_{\mathrm{x}}$ component is missing.
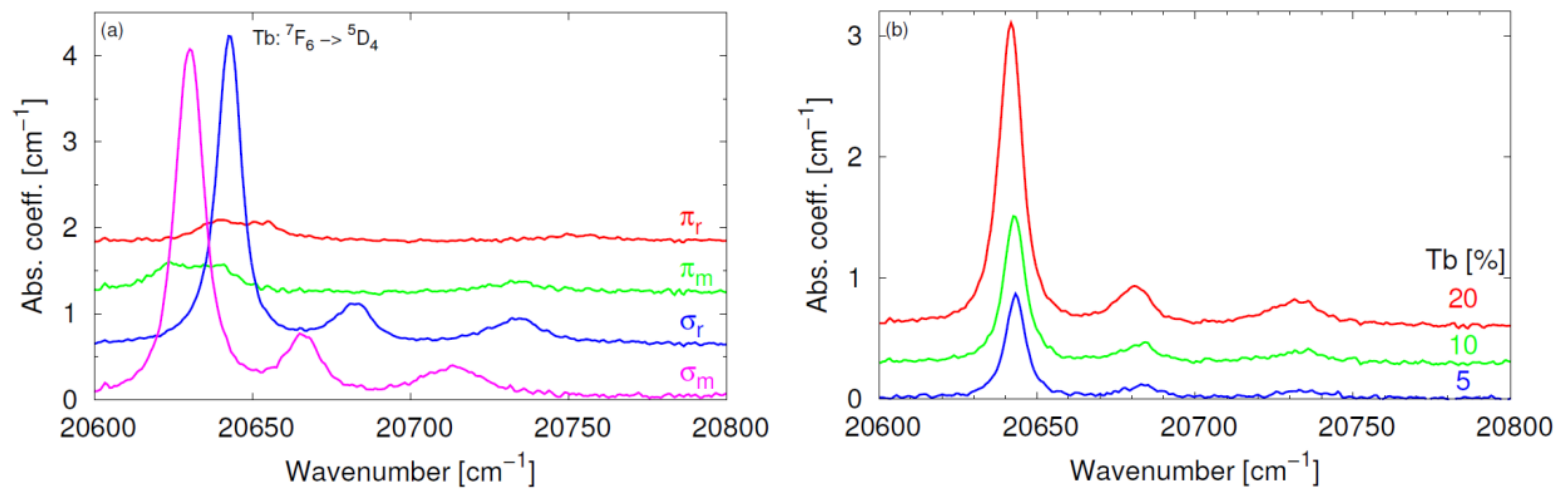

Fig. 2. Polarization $\left(\pi\right.$ and $\sigma$ ) dependence of the $\mathrm{Tb}:{ }^{7} \mathrm{~F}_{6}-{ }^{5} \mathrm{D}_{4}$ transition in doped GAB crystals of both modifications (No. 4-5, r: rhombohedral, m: monoclinic phase as discussed in the text) (a). Absorption spectra of GAB:Tb crystals (No. 1-3) as a function of the $\mathrm{Tb}$ concentration (b).

Among the electronic transitions of $\mathrm{Tb}^{3+}$ ions only the ${ }^{5} \mathrm{D}_{4}$ multiplet falls into the visible spectral range. The ${ }^{5} \mathrm{D}_{4}$ multiplet due to the Coulomb repulsion between $4 \mathrm{f}$ electrons and the spin-orbit interactions is in the range of $20600-20800 \mathrm{~cm}^{-1}(485-480 \mathrm{~nm})$ above the ${ }^{7} \mathrm{~F}_{6}$ ground state. A slight shift of these band positions by at about $12-19 \mathrm{~cm}^{-1}$ can be observed in Fig. 2a for both polarizations due to the change from the rhombohedral to the monoclinic structure (crystals No. 4-5). In order to exclude the concentration change of the $\mathrm{Tb}^{3+}$ ions as the origin of the shift, the absorption bands have also been measured as a function of the $\mathrm{Tb}$ 
content (see Fig. 2b). The change of the concentration of $\mathrm{Tb}^{3+}$ ions in a relatively wide range (5-20 mol\%, No. 1-3) induces only a $1-2 \mathrm{~cm}^{-1}$ shift of the main absorption band corresponding to the ${ }^{7} \mathrm{~F}_{6}-{ }^{5} \mathrm{D}_{4}$ transition. This result supports the assumption that the difference of the band positions, present in the symmetry modifications, essentially originates from the specific environments of $\mathrm{Tb}^{3+}$ ions in the $\mathrm{GAB}$ crystal lattices changing both the crystal field (CF) symmetry and the crystal field strength. The common shift of all lines in the ${ }^{7} \mathrm{~F}_{6}-{ }^{5} \mathrm{D}_{4}$ transition indicates that not only the crystal field splitting pattern is altered within the ${ }^{5} \mathrm{D}_{4}$ multiplet, but also the barycenter of the ${ }^{5} \mathrm{D}_{4}$ multiplet is shifted due to changes of the Coulomb and spin-orbit interactions.

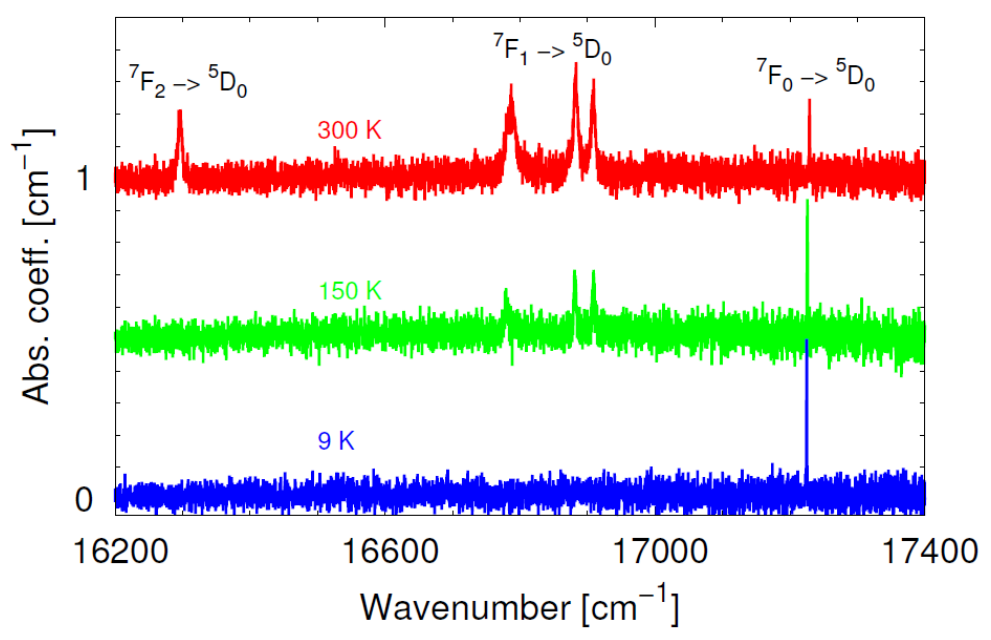

Fig. 3. Temperature dependence of the absorption bands of the ${ }^{7} \mathrm{~F}_{0,1,2}-{ }^{5} \mathrm{D}_{0}$ transitions of $\mathrm{Eu}^{3+}$ in monoclinic GAB:Tb,Eu (No. 5) crystal.

For the assignation of the absorption bands to the electronic levels of the $\mathrm{Eu}^{3+}$ ions temperature dependent absorption measurements were also performed. The absorption spectra of $\mathrm{Eu}^{3+}$ ions in the rhombohedral GAB crystal (No. 4) show similar behavior as already measured in [10]. One can conclude from these measurements that the electronic transitions from the ${ }^{7} \mathrm{~F}_{0}$ ground state and the thermally populated ${ }^{7} \mathrm{~F}_{1}$ and ${ }^{7} \mathrm{~F}_{2}$ multiplets of europium can be detected at room temperature $[9,10]$. However, a shift and splitting of the bands by values in the range of $4-30 \mathrm{~cm}^{-1}$ depending on the electronic transitions were observed in the absorption spectra of the monoclinic structure due to the symmetry reduction and its consequences for the Coulomb, spin-orbit and crystal field interactions. E.g. the crystal field splitting of the ${ }^{7} \mathrm{~F}_{1}$ hot transition results in three absorption bands in the high temperature spectra as can be seen in Fig 3. 

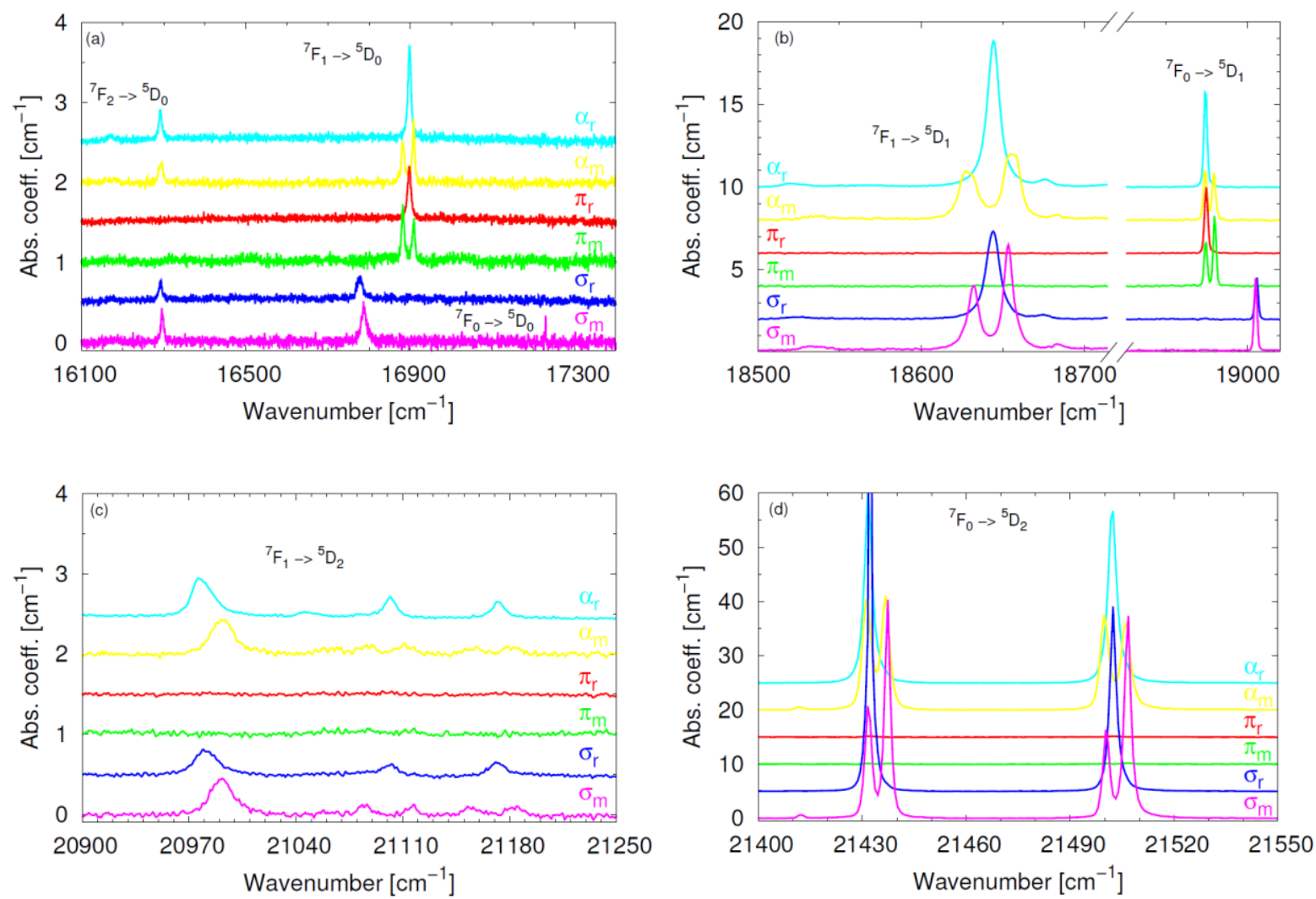

Fig. 4. Absorption spectra corresponding to the electronic transitions from the ${ }^{7} \mathrm{~F}_{0,1,2} \mathrm{CF}$ levels to the ${ }^{5} \mathrm{D}_{0,1,2} \mathrm{CF}$ levels (a-d) of the $\mathrm{Eu}^{3+}$ ion with $\alpha, \pi$ and $\sigma$ polarizations in both modifications, measured at room temperature (Samples No. 4-5).

Fig. 4. shows the polarization dependent absorption spectra of $\mathrm{Eu}^{3+}$ ions in both rhombohedral and monoclinic GAB crystals at room temperature. The electronic transitions from the ${ }^{7} \mathrm{~F}_{0,1,2}$ multiplets to ${ }^{5} \mathrm{D}_{0}$ singlet can be observed in the $16100-17300 \mathrm{~cm}^{-1}$ wavenumber range (Fig. 4a.). The forbidden band of the ${ }^{7} \mathrm{~F}_{0}{ }^{5} \mathrm{D}_{0}$ transition, that is invisible in rhombohedral symmetry, evolves into a $\sigma$ polarized absorption spectrum of the monoclinic GAB crystal. The small change of the local symmetry makes it observable with a magnetic dipole behavior (Table 2). In both modifications the transitions from the ${ }^{7} F_{1}$ and ${ }^{7} F_{2}$ levels have magnetic and electric dipole character, respectively. Since ${ }^{5} \mathrm{D}_{0}$ is a singlet every splitting in the absorption spectra in this range is a consequence of the crystal field splitting of the fundamental ${ }^{7} \mathrm{~F}_{1,2}$ multiplets in the monoclinic modification. So the splitting of the band by at about $16900 \mathrm{~cm}^{-1}$ into two bands at $16880 \mathrm{~cm}^{-1}$ and $16910 \mathrm{~cm}^{-1}$ corresponds to the break-up of the doubly degenerated ${ }^{7} \mathrm{~F}_{1}$ multiplet.

Absorption bands corresponding to transitions to the ${ }^{5} \mathrm{D}_{1}$ level have been investigated in the 18500-19000 $\mathrm{cm}^{-1}$ wavenumber range. The magnetic dipole feature of the ${ }^{7} \mathrm{~F}_{0}-{ }^{5} \mathrm{D}_{1}$ 
transition can be clearly seen in the polarization dependent absorption spectra (see Fig. $4 \mathrm{~b}$ and Table 2). Additionally, a small splitting of the band at $18974 \mathrm{~cm}^{-1}$ by at about $6 \mathrm{~cm}^{-1}$ shows the loss of the degeneracy of the corresponding ${ }^{5} \mathrm{D}_{1} \mathrm{CF}$ level due to symmetry reduction. The hypersensitive ${ }^{7} \mathrm{~F}_{1}-{ }^{5} \mathrm{D}_{1}$ transition shows electric dipole behavior and a similar splitting by at about $30 \mathrm{~cm}^{-1}$ as it was already seen for the splitting of one of the ${ }^{7} \mathrm{~F}_{1} \mathrm{CF}$ levels in the ${ }^{7} \mathrm{~F}_{1}-{ }^{5} \mathrm{D}_{0}$ transition.

The absorption bands of the ${ }^{7} \mathrm{~F}_{0,1}-{ }^{5} \mathrm{D}_{2}$ transitions were observed in the $20900-21550 \mathrm{~cm}^{-1}$ wavenumber range (Fig. $4 \mathrm{c}-\mathrm{d}$ ). Although the ${ }^{7} \mathrm{~F}_{1}-{ }^{5} \mathrm{D}_{2}$ transition gives only low intensity absorption bands the splitting by at about $30 \mathrm{~cm}^{-1}$ corresponding to the ${ }^{7} \mathrm{~F}_{1} \mathrm{CF}$ level is clearly observable (Fig. 4c). The most intense absorption bands in the spectra correspond to the ${ }^{7} \mathrm{~F}_{0}$ ${ }^{5} \mathrm{D}_{2}$ transition which shows electric dipole behavior (Fig. $4 \mathrm{~d}$ and Table 2). Because of the single ground state the absorption band positions clearly correspond to the structure of the excited ${ }^{5} \mathrm{D}_{2}$ multiplet. It was found that the degenerate ${ }^{5} \mathrm{D}_{2} \mathrm{CF}$ levels were split by at about 5$7 \mathrm{~cm}^{-1}$ due to the symmetry reduction.

Assigning the band positions in the room temperature absorption measurements and taking into consideration the polarization dependence one can determine the energies of the ${ }^{7} \mathrm{~F}_{0,1,2}$ and ${ }^{5} \mathrm{D}_{0,1,2} \mathrm{CF}$ levels. The resulting electronic energy level diagrams for both symmetry variations are shown in Fig. 5. These results can be used for crystal field calculations in a unique way because the crystal lattice and also the environment of the rare earth ions differ only nearly imperceptibly in these two modifications. According to [20] the energy levels of ${ }^{5} \mathrm{D}_{1}$ and ${ }^{7} \mathrm{~F}_{1}$ multiplets of $\mathrm{Eu}^{3+}$ ion behave similarly in different environments. It was shown theoretically that the ratio of the splitting of these two terms has to be 0.298 independent from crystal symmetry, however, the experimental values usually differ (e.g. 1/5 in [11]) from this prediction which was interpreted by intermediate coupling or J-mixing. The ratios for the rhombohedral and monoclinic GAB crystals are 0.24 and 0.258 , respectively. These values indicate that the LS coupling model describes the $\mathrm{Eu}^{3+}$ ions better in the monoclinic structure than in the rhombohedral one. 


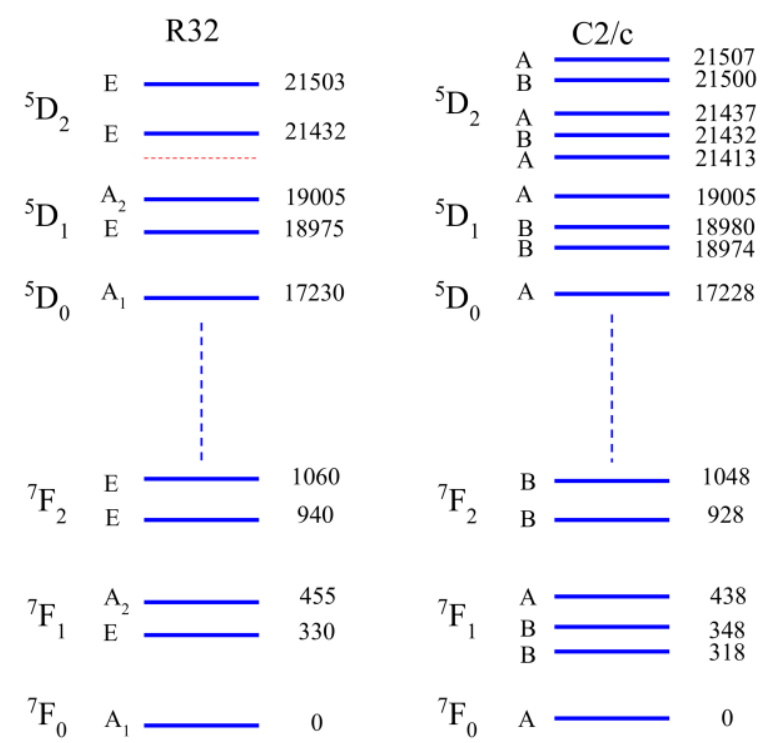

Fig. 5. The lower part of the electronic energy levels of $\mathrm{Eu}^{3+}$ ion determined from absorption measurements at room temperature for both rhombohedral and monoclinic modifications, the energy values are given in $\mathrm{cm}^{-1}$.

\section{Conclusions}

Electronic transitions of the $\mathrm{Eu}^{3+}, \mathrm{Tb}^{3+}$ and $\mathrm{Gd}^{3+}$ rare earth ions in doped $\mathrm{GAB}$ crystals with monoclinic $(C 2 / c)$ and rhombohedral $(R 32)$ structure were successfully identified using temperature and concentration dependent absorption measurements in the $16000-32000 \mathrm{~cm}^{-1}$ wavenumber range. The absorption bands of $\mathrm{Tb}^{3+}$ ions shifted less than $20 \mathrm{~cm}^{-1}$ due to the symmetry change. The electronic transitions of the $\mathrm{Eu}^{3+}$ ions between the ${ }^{7} \mathrm{~F}_{0,1,2}$ and ${ }^{5} \mathrm{D}_{0,1,2}$ levels were also successfully identified by additional polarization dependent absorption measurements at room temperature. The appearance of the strongly forbidden ${ }^{7} \mathrm{~F}_{0}{ }^{5} \mathrm{D}_{0}$ transition and the splitting of the degenerate levels due to symmetry reduction make detailed crystal field calculations most feasible. The ratios of the splitting of the ${ }^{5} \mathrm{D}_{1}$ and ${ }^{7} \mathrm{~F}_{1}$ multiplets show that the LS coupling scheme is better for the description of the $\mathrm{Eu}^{3+}$ ions in the monoclinic structure than in the rhombohedral case.

\section{Acknowledgement}

This work was supported by the Janos Bolyai research fellowship of the Hungarian Academy of Sciences and the Hungarian Scientific Research Fund (OTKA No. K83390). 


\section{References}

[1] E. Beregi, I. Sajó, K. Lengyel, P. Bombicz, M. Czugler, I. Földvári, J. Crys. Growth 351 (2012) 72-76.

[2] A. A. Balmann, The American Mineralogist 47, November-December, (1962) 1380-1383.

[3] A. D. Mills, Inorganic Chemistry 1, (1962) 960-961.

[4] N. I. Leonyuk, L. I. Leonyuk, Prog. Crystal Growth and Charact. 31 (1995) 179.

[5] A. Mayolet, W. Zhang, P. Martin, B. Chassigneux, J.C. Krupa, J. Electroch. Soc. 143 (1) (1996) 330.

[6] E. C. Fuchs, C. Sommer, F. P. Wenzl, B. Bitschnau, A. H. Paulitsch, A. Mühlanger, K. Gatterer, Materials Science and Engineering B 156 (2009) 73-78.

[7] A. A. F. Lagerwey, G. Blasse, Chem. Phys. Lett. 31 (1975) 27.

[8] R. D. Peacock, Chem. Phys. Lett. 35 (1975) 420.

[9] R. Kuroda, S. F. Mason and C. Rosini, J. Chem. Soc., Faraday Trans. 277 (1981) 2125.

[10] C. Görller-Walrand, E. Huygen, K. Binnemans and L. Fluyt, J. Phys.: Condens. Matter. 6 (1994) 7797.

[11] K. Binnemans, Coordination Chemistry Reviews 295 (2015) 1-45.

[12] K. Binnemans, K. Van Herck, C. Görller-Walrand, Chemical Physics Letters 266 (1997) 297-302.

[13] R. D. Peacock, Structure Bonding, 22 (1975) 83-122.

[14] P. A. Plachinda and E. L. Belokoneva, Cryst. Res. Technol. 43 (2008) 157-165.

[15] E. L. Belokoneva, T. I. Timchenko, Soviet Physics Crystallography 28 (1983) 658-661.

[16] B. B. Zvyagin and E. L. Belokoneva, Soviet Physics Crystallography 29 (1984) 173-176.

[17] E. Beregi, E. Hartmann, L. Malicskó, J. Madarász, Crystal Research and Technology 34 (1999) 641.

[18] J. García Solé, L.E. Bausá and D. Jaque, “An Introduction to the Optical Spectroscopy of Inorganic Solids", 2005 John Wiley \& Sons Ltd, The Atrium, Southern Gate, Chichester, West Sussex PO19 8SQ, England [19] M.S. Dresselhaus, G. Dresselhaus, A. Jorio, "Group Theory: Application to the Physics of Condensed Matter", 2008 Springer-Verlag Berlin Heidelberg [20] B. R. Judd, Proc. R. Soc. Lond. A 228 (1955) 120-128 